\title{
On the High-Temperature Creep Behaviour and Substructures in Alpha-Iron Single Crystals
}

\author{
By S. Karashima*, T. Iikubo*, and H. Oikawa*
}

\begin{abstract}
Creep tests of the (001)[110] iron single crystals were done in the temperature range from $600^{\circ} \mathrm{C}$ to $880^{\circ} \mathrm{C}$ under normal stress from 0.5 to $2.5 \mathrm{~kg} / \mathrm{mm}^{2}$. Creep behaviour of the single crystal was essentially the same as that of polycrystalline iron at a stress larger than $0.7 \mathrm{~kg} / \mathrm{mm}^{2}$; grain boundary has a minor role in the present experimental condition. Subgrains were formed during creep as in the case of molybdenum single crystals. Many screw dislocations were observed within subgrains. Subgrain size was almost inversely proportional to creep stress and the dislocation density within subgrains was proportional to creep stress, while both of them were independent of creep temperature.

(Received September 18, 1971)
\end{abstract}

\section{Introduction}

A large number of investigations on high-temperature creep have been reported and the factors affecting hightemperature strength of materials have been considered to some extent ${ }^{(1)}$. Elementary processes of creep deformation have also been discussed ${ }^{(2)}$ on the basis of dislocation motion. The materials used in these works, however, are usually polycrystalline. Hence, some difficulties always arise in discussing creep mechanisms because of the complexity of stress conditions in the specimens and of structures resulting from the deformation. Moreover, at high temperature the deformation of grains is always associated with the deformation at grain boundaries. Therefore, comprehensive studies on single crystals are necessary to discuss dislocation behaviour during high-temperature creep deformation. No extensive investigations have been reported on hightemperature creep behaviour of iron single crystals, though iron is one of the most important materials for practical use.

In this paper creep behaviour of alpha-iron single crystals over a wide range of stress and temperature is reported to give fundamental data in discussing creep mechanisms. The microstructures after creep deformation of the iron single crystals are also examined with etch-pit technique and with transmission electron microscopy to make clear the relation between creep behaviour and dislocation structures.

\section{Experimental Procedure}

The material used was an iron single crystal sheet of $1 \mathrm{~mm}$ in thickness, the surface of which was a (001)plane. The sheet was made by strain-annealing of vacuum-melted electrolytic iron ${ }^{(3)}$. Specimens with a gauge length of $15 \mathrm{~mm}$ and a width of $5 \mathrm{~mm}$ were prepared from the sheet so that the [110]-direction was

* Department of Materials Science, Faculty of Engineering, Tohoku University, Sendai, Japan.

(1) For example, O.D. Sherby and P. M. Burke : Progress Mat. Sci, Pergamon Press, Vol. 13, (1968), p. 323.

(2) For example, T. Watanabe and S. Karashima : Trans. JIM, 11 (1970), 159.

(3) T. Takeuchi : Trans. JIM, 7 (1966), 1. parallel to the tensile axis ${ }^{\dagger}$. They were decarburized by annealing at $720^{\circ} \mathrm{C}$ for $40 \mathrm{hr}$ in wet-hydrogen and then for $18 \mathrm{hr}$ in purified hydrogen.

Constant-stress tensile creep tests were done in argon atmosphere at temperatures between $600^{\circ}$ and $880^{\circ} \mathrm{C}$ $\left(0.48 \sim 0.64 T_{m}\right)$ under a normal stress ranging from 0.5 to $2.5 \mathrm{~kg} / \mathrm{mm}^{2}$. The testing temperatures were maintained constant within $\pm 2^{\circ} \mathrm{C}$ of the reported values. Creep elongation was continuously recorded using a linear differential transformer and was also measured directly with a dial-gauge reading to $1 / 100 \mathrm{~mm}$.

After creep tests the specimens were cooled rapidly under load to preserve the deformation structures. The sub-boundaries in the deformed $(001)[110]$ crystals were revealed on the (001)-surface by the following reagent ${ }^{(4)}$ : $\mathrm{FeCl}_{3} \cdot 6 \mathrm{H}_{2} \mathrm{O}(20 \mathrm{~g})$, ethyl alcohol $(40 \mathrm{~mL})$, water $(40 \mathrm{~mL})$, and $\mathrm{CuCl}_{2} \cdot 6 \mathrm{H}_{2} \mathrm{O}$ (a few mg). Dislocation arrangements within subgrains were examined by means of etch-pit technique using a potentiostat ${ }^{(5)}$ and by transmission electron microscopy with an electron microscope SMH$5 \mathrm{~A}$ operating at $500 \mathrm{kV}$.

\section{Results and Discussion}

Slip bands formed on the (001)- and (1I 0 )-planes during creep deformation are shown in Photo. 1. It is clearly seen in Photo. 1 (a) that slip bands are very wavy with a general direction of $[1 \overline{1} 0]$. It was confirmed that the active slip systems were $(\overline{I I} 2)[111]$ and (112) [111 $]$ in these $(001)[110]$ crystals. These results are in good agreement with those obtained in tensile deformation of alpha-iron single crystals ${ }^{(6)}$ at temperatures of $25^{\circ}$ to $900^{\circ} \mathrm{C}$ and in high-temperature creep of molybdenum single crystals $^{(7)}$. Because of the cooperative action of the two slip systems, little crystal rotation occurred during creep deformation. Therefore, no complexity was involved in the analysis of creep data.

$\dagger$ The single crystals are named the (001)[110] crystals in this papar.

(4) T. Takeuchi : J. Phys. Soc. Japan, 20 (1965), 942.

(5) T. Shibata, M. Hachinohe and T. Takeyama : J. Japan Inst. Metals, 33 (1969), 1320.

(6) S. Ikeda : J. Phys. Soc. Japan, 27 (1969), 1564.

(7) A.H.Clauer, B.A.Wilcox and J.P. Hirth : Acta Met., 18 (1970), 367. 


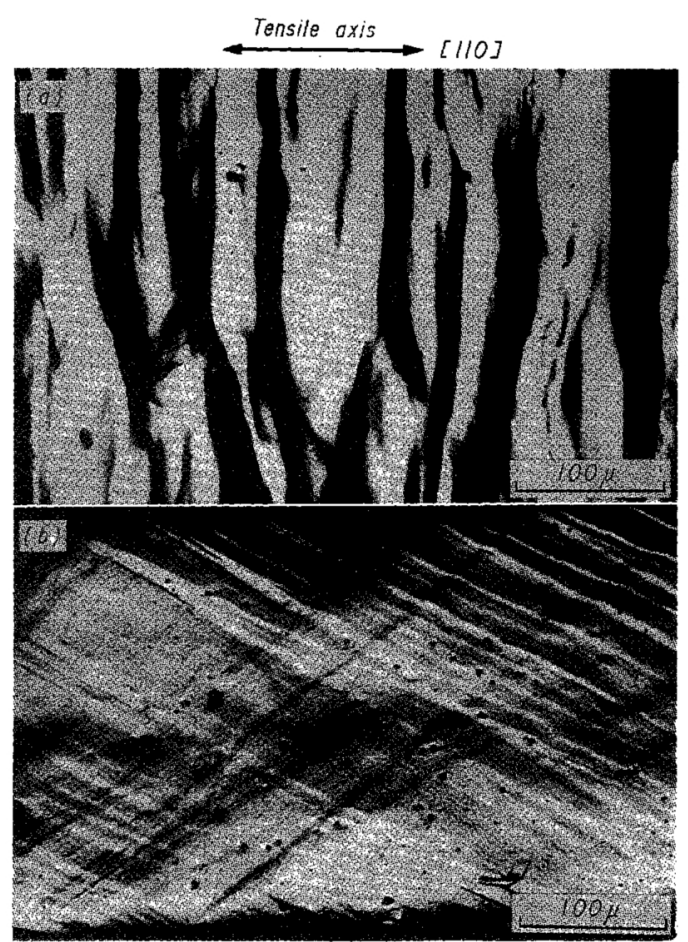

Photo. 1 Slip bands after $40 \%$ creep strain at $1.4 \mathrm{~kg} / \mathrm{mm}^{2}$ and $700^{\circ} \mathrm{C}$. (a) (001) view, (b) (110) view

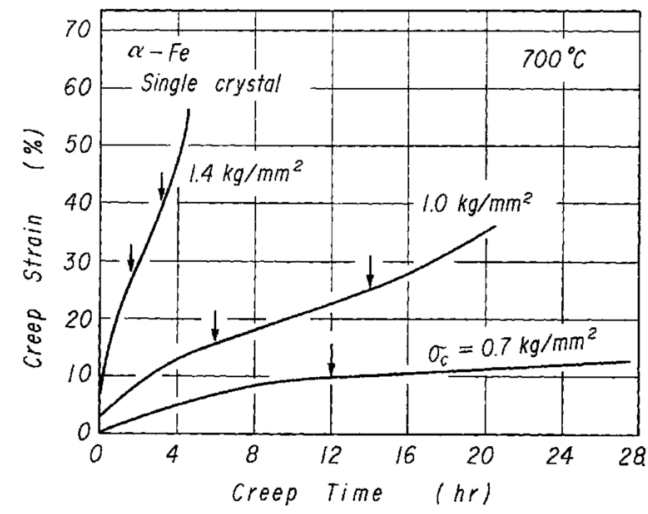

Fig. 1 Typical creep curves of the $(001)[110]$ single crystals.

\section{Creep curves}

Typical creep curves obtained are shown in Fig. 1 . As obviously seen in Fig. 1, the usual creep curve consisting of transient, steady-state and accelerating stages was obtained at a stress larger than $0.7 \mathrm{~kg} / \mathrm{mm}^{2}$. At a stress less than $0.7 \mathrm{~kg} / \mathrm{mm}^{2}$ at $700^{\circ} \mathrm{C}$, however, an unusual creep behaviour was observed. The anomaly of creep behaviour at a low stress has been reported elsewhere ${ }^{(8)}$.

Recently, Clauer et al. (7) have found out the absence of steady-state in molybdenum single crystals having the same orientation as the present crystals. Clauer ${ }^{(9)}$ has also reported that a creep curve just like that of the single crystals can be obtained in a coarse-grained, hydrogen-annealed specimen, while the usual creep curve consisting of the three stages is observed in the

(8) T. Iikubo, H. Oikawa and S. Karashima: Scripta Met., 5 (1971), 837.

(9) A.H. Clauer : Ph.D. Dissertation, The Ohio State Univ. (1968). as-received (non-decarburized) state. These results suggest that the occurrence of the steady-state is related to the existence of impurities. However, the specimen used in the present work was decarburized for a long time. Therefore, the difference between the results by Clauer et al. ${ }^{(7)}$ and by the present authors should be ascribed to other effects than those of impurities.

Creep rates of the single crystals and polycrystalline iron $^{(10)}$ under the same creep condition are shown in Fig. 2 as a function of strain. Creep rate during transient stage of the single crystals was remarkably larger than that of polycrystalline iron. On the other hand, the steady-state creep rates were almost the same. Strain from the beginning of the secondary stage, $\varepsilon_{s}$, up to the beginning of the tertiary stage, $\varepsilon_{t r}$, are shown by arrows in Fig. 2, respectively. It is clearly seen that $\varepsilon_{s}$ and $\varepsilon_{t r}$ of the single crystals were larger than those of polycrystalline iron. The results would show that it takes a longer time for the single crystals to reach an equilibrium dislocation arrangement as Clauer et al. have suggested $^{(7)}$.

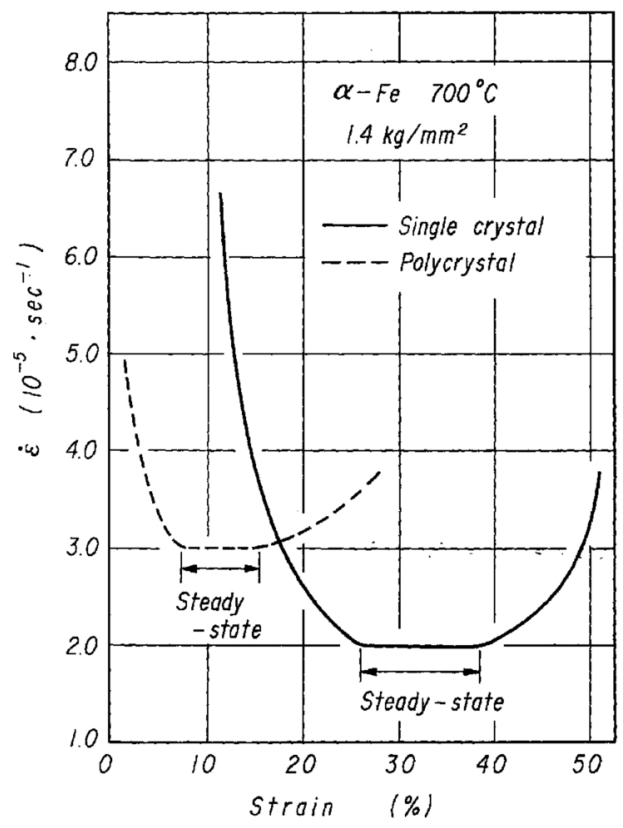

Fig. 2 Creep rates as a function of strain.

\section{Steady-state creep rates}

Temperature dependence of $\dot{\varepsilon}_{s}$ in the $(001)$ [110] single crystal is shown in Fig. 3, together with data on polycrystalline iron ${ }^{(10)}$ whose average grain diameter was about $80 \mu$. Taking the temperature dependence of $E_{T}{ }^{(11)}$ into consideration, creep stress, $\sigma_{c}$, at each temperature is chosen so that $\sigma_{c} / E_{T}$ is constant, where $E_{T}$ is the elastic modulus at the testing temperature. As seen in the figure, creep rate of the (001) [110] single crystal is almost the same as that of polycrystalline specimen. Similar results have been reported on an $\mathrm{Fe}-\mathrm{Si}$ alloy ${ }^{(12)}$. The results indicate that grain boundary sliding has a minor role in this case.

(10) S. Karashima, H. Oikawa and T. Watanabe : Trans. Met Soc. AIME, $242(1968), 1703$.

(11) W. Köster : Z. Metallk., 39 (1948), 1.

(12) A. Solomon and W. D. Nix : Acta Met., 18 (1970), 863. 


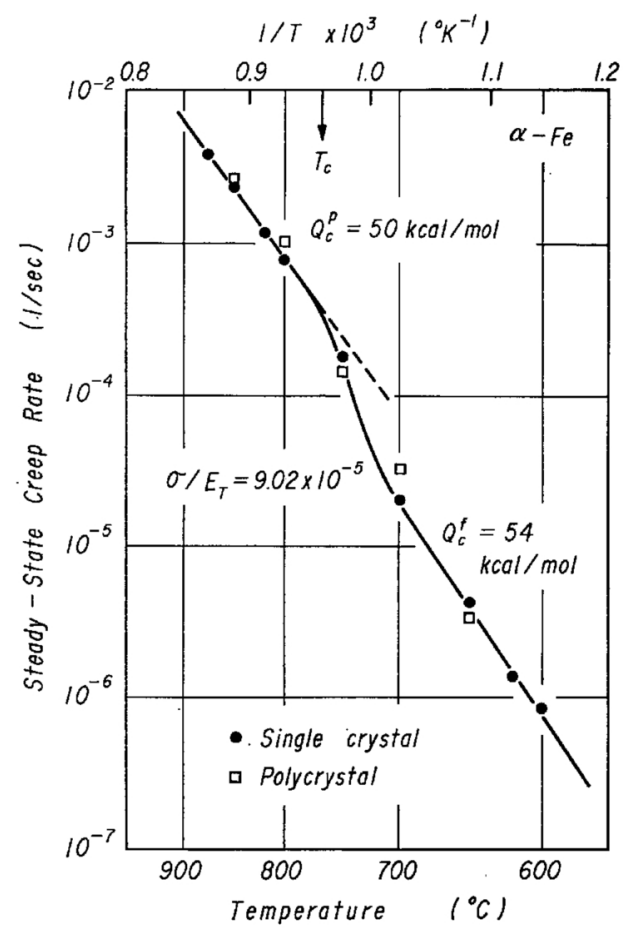

Fig. 3 Temperature dependence of steady-state creep rate, $\dot{\varepsilon}_{s}$.

The change in creep rates in the vicinity of the Curie temperature, i.e., about $770^{\circ} \mathrm{C}$, is due to the magnetic effects as has been reported in several metals ${ }^{(13) \sim(16)}$ and alloys ${ }^{(10)(17) \sim(22)}$.

In the ferromagnetic and paramagnetic temperature regions, the temperature dependence of steady-state creep rates can be represented by the following equation.

$$
\dot{\varepsilon}_{s}=A f(\sigma / E) \exp \left(-Q_{c} / R T\right) .
$$

The activation energy for steady-state creep, $Q_{c}$, was determined from the slope of the straight line in Fig. 3. The results show $Q_{c}^{p}=50 \pm 2.0 \mathrm{kcal} / \mathrm{mol}$ for the paramagnetic temperature region and $Q_{c}^{f}=54 \pm 1.8 \mathrm{kcal} / \mathrm{mol}$ for the ferromagnetic temperature region. They are smaller than that for self-diffusion, $Q_{s d}$, by about $7 \sim 8$ $\mathrm{kcal} / \mathrm{mol}^{(23)}$.

On the other hand, the activation energy for creep, $Q_{c}$, in eq. (1) is described as follows :

$$
Q_{c}=Q_{s d}-A^{*} b \tau^{*}
$$

where $A^{*}$ is the activation area for creep, $b$ the Burgers vector, and $\tau^{*}$ the effective shear stress. On the assumption that $\tau^{*} / \tau=0.35^{(2)}, A^{*}$ can be estimated from eq. (2); $A^{*} \simeq 1300 b^{2}$ for $\sigma=1.4 \mathrm{~kg} / \mathrm{mm}^{2 \dagger}$. It is about the same as that obtained by stress-relaxation

(13) Y.Ishida, C. Y. Cheng and J.E.Dorn : Trans. Met. Soc. AIME, 236 (1966), 964.

(14) J. Cadek, M. Pahutova, K. Ciha and T. Hostinsky : Acta Met., $17(1969), 803$.

(15) P.R. Landon, J. L. Lytton, L.A. Shepard and J.E.Dorn : Trans. ASM, 51 (1959), 900.

(16) E. C. Norman and S. A. Duran : Acta Met., 18 (1970), 723.

(17) C.Y. Cheng, A. Karim, T.G. Langdon and J.E. Dorn : Trans. Met. Soc. AIME, 242 (1968), 890.

(18) A. Karim : Canad. J. Phys., 46 (1968), 2425.

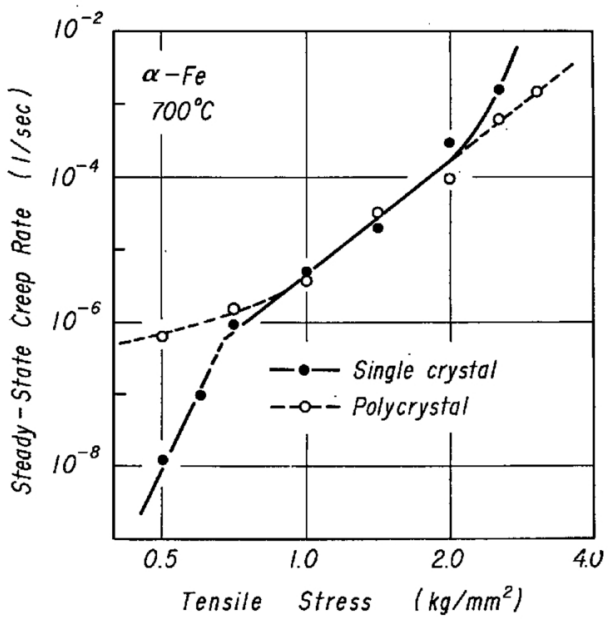

Fig. 4. Stress dependence of steady-state creep rate, $\dot{\varepsilon}_{s}$.

tests during creep of polycrystalline iron ${ }^{(2)}$, that is about $1000 b^{2}$.

The stress dependence of $\dot{\varepsilon}_{s}$ at $700^{\circ} \mathrm{C}$ is shown in Fig. 4, together with data on polycrystalline iron(10). As shown in the figure, creep rates in the single crystals are almost the same as those in polycrystalline iron over a stress range of $0.7 \sim 2.0 \mathrm{~kg} / \mathrm{mm}^{2}$. The difference between the single crystal and the polycrystalline iron in a low stress region (less than $0.7 \mathrm{~kg} / \mathrm{mm}^{2}$ ) has been ascribed to the influence of grain boundaries ${ }^{(8)}$. Although the origin of the deviation of creep rates at a stress larger than $2.0 \mathrm{~kg} / \mathrm{mm}^{2}$ is not certain, the rapid increase in $\dot{\varepsilon}_{s}$ in the single crystals may correspond to the transition from "range 2 " to "range 3 " proposed by Sherby and Burke ${ }^{(1)}$.

\section{Substructures}

\section{(1) Sub-boundaries}

Effects of sub-structures, particularly those of subboundaries, on creep deformation have recently come to receive increasing attention and many papers have been published on the contributions of sub-boundaries to high-temperature creep deformation. Cuddy ${ }^{(24)}$ has discussed the relation between subgrain size and internal stress. Watanabe and Karashima ${ }^{(2)}$ have pointed out an important role of sub-boundaries in high-temperature creep. They have supposed in the super-jog model that elementary jogs are produced on a screw dislocation when it leaves sub-boundaries.

Substructures revealed by usual etching are given in Photo. 2, from which it is evident that subgrains extending in the direction of slip bands are formed during high-temperature creep. Similar long subgrains were observed in molybdenum single crystals ${ }^{(25)}$. The

+ Shear stress is calculated from the relation $\tau=\alpha / 2$.

(19) Y. Imai and T. Murata : J. Japan Inst. Metals, 29 (1965), 1053.

(20) A. Fuchs and B. Ilschner : Acta Met., 17 (1969), 701.

(21) S. Karashima, T. Motomiya and H. Oikawa: Tech. Repts., Tohoku Univ., 33 (1968), 193.

(22) H. Oikawa, K. Oguchi and S. Karashima : Scripta Met., 5 (1971), 825.

(23) F.S.Buffington, K. Hirano and M. Cohen : Acta Met., 9 (1961), 434.

(24) L. J. Cuddy : Met. Trans., 1 (1970), 395.

(25) A. H. Clauer, B. A. Wilcox and J.P.Hirth : Acta Met., 18 (1970), 381. 
size of the subgrains formed during steady-state creep measured in the direction of [110] is shown in Fig. 5 as a function of creep stress and temperature. As can be seen in the figure, the subgrain size is not dependent on creep temperature but on creep stress; the higher the creep stress, $\sigma_{c}$, the finer the subgrain size, $d$. The relationship $d=\mathrm{A} \sigma_{c}^{-n}$ was obtained, $n$ being nearly equal

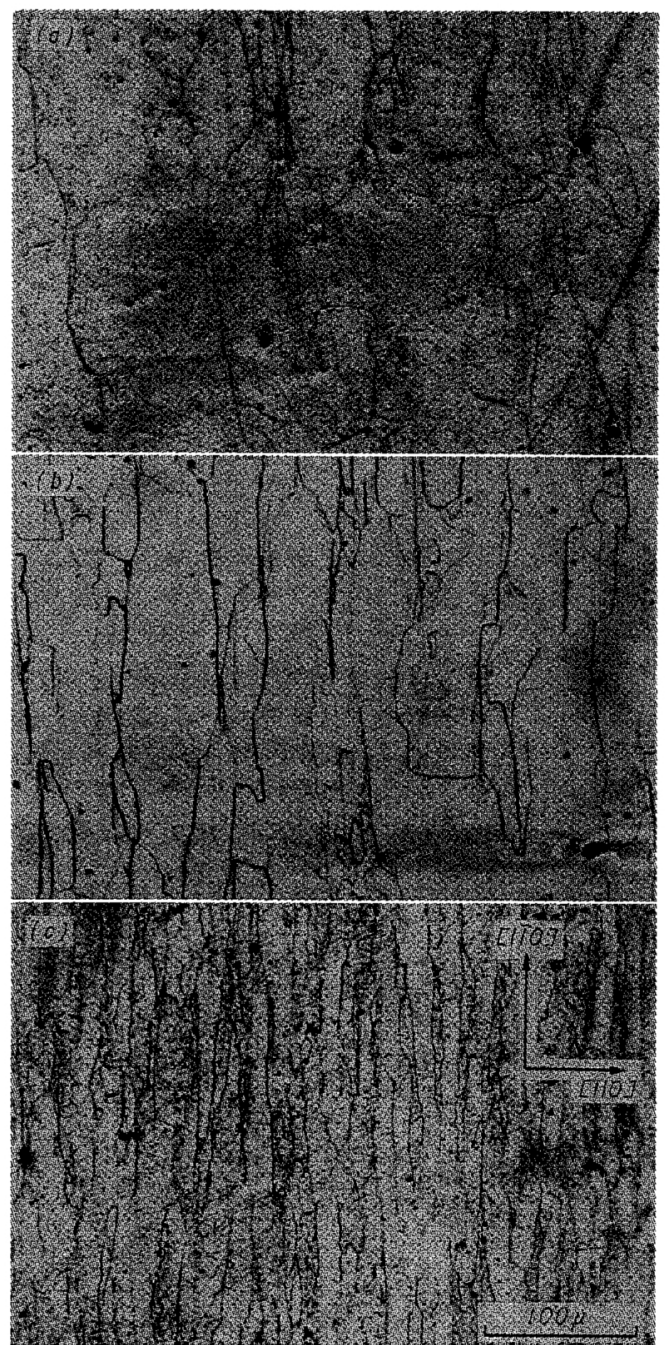

Photo. 2 Subgrains formed due to creep at $700^{\circ} \mathrm{C}$ and (a) 0.7 $\mathrm{kg} / \mathrm{mm}^{2}$, (b) $1.4 \mathrm{~kg} / \mathrm{mm}^{2}$ and (c) $2.5 \mathrm{~kg} / \mathrm{mm}^{2}$.

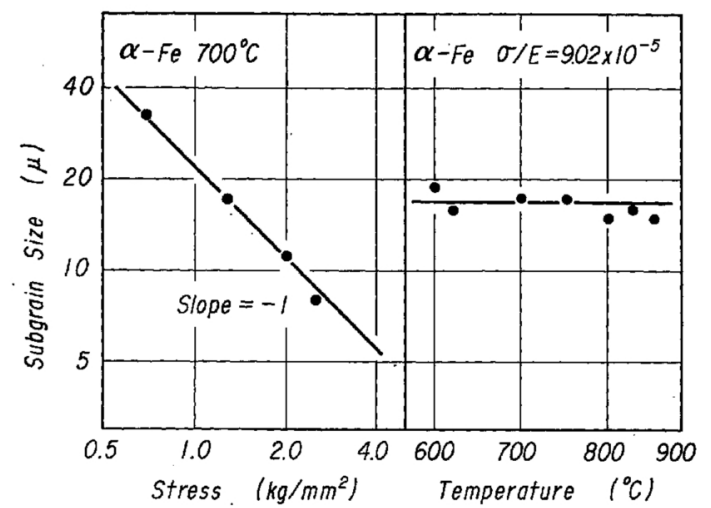

Fig. 5 Subgrain size due to steady-state creep measured in the direction parallel to the tensile axis, $[110]$, as a function of creep stress (a) and temperature (b).

(26) I. S. Servi and N. J. Grant : Trans. AIME, 191(1951), 909.

(27) C. R. Barrett, J. L. Lytton and O. D. Sherby : Trans. Met. Soc. AIME, 239 (1967), 170. to unity. The same value of $n$ has been reported on several polycrystalline pure metals ${ }^{(26) \sim(29)}$.

\section{(2) Dislocation arrangement}

An example of micrographs showing the distribution of etch-pits on the (001)-plane after creep is given in Photo. 3. Two regions extending in the direction parallel to slip bands appear alternatively ; one (region A) consists of relatively fine structure and the other (region B) of coarse structure. In each region, the substructure was made up of subgrains with relatively low dislocation density surrounded by sub-boundaries of high dislocation density. Similar substructures have been reported on molybdenum single crystals of the same orientation ${ }^{(25)}$.

In contrast to semi-micro structures shown in Photo. 2 , the subgrains in region $B$ revealed by the etch-pit technique (Photo. 3) are nearly equi-axed. The difference in structures revealed by usual etching and by etch-pit technique suggests that the usual etching reagent attacks preferentially a particular type of dislocation arrays. Density of etch-pits not associated with sub-boundaries is given in Fig. 6 as a function of creep temperature and stress ; etch-pit density is not dependent on creep temperature but on creep stress. In Fig. 6 , the dislocation density measured by transmission electron microscopy is also given. Dislocation density within subgrains is linearly proportional to creep stress. Similar stress dependence has been reported on polycrystalline iron by the present authors ${ }^{(30)}$.

A typical dislocation structure after creep, obtained by transmission electron microscopy, is reproduced in Photo. 4. Sub-boundaries extending in the direction of [ $1 \overline{1} 0]$ were of ten observed, as in the case of molybdenum single crystals ${ }^{(25)}$. According to Clauer et al., these sub-boundaries were [111]-tilt boundaries in molybdenum. This may be also the case for the present iron crystals. However, the present results on the character of dislocations within subgrains differ remarkably from the observations in molybdenum. They ${ }^{(25)}$ have reported that few dislocations exist within subgrains and most of the dislocations are of the edgetype. On the other hand, as clearly seen in Photo. 4, many dislocations are observed within subgrains, some of them being screw dislocations. One of the reasons why such a difference was obtained may be the difference in specimen thickness. It is very likely that some dislocations were lost in their case as a result of the use of thin specimens for transmission electron microscopy. The extreme value of low dislocation density $\left(2 \times 10^{6} \mathrm{~cm}^{-2}\right)$ reported by them seems to be caused for the same reason. In the present experiments care was taken using a $500 \mathrm{kV}$ electron microscope so that the structures of specimens with a thickness as large as possible might be photographed. However,

$\uparrow$ Details of dislocation structures will be published elsewhere ${ }^{(31)}$.

(28) A. Goldberg : J. Iron Steel Inst., 204 (1966), 268.

(29) R. W. Guard : Creep and Recovery, ASM, (1957), p. 251.

(30) S. Karashima, T. Iikubo, T. Watanabe and H. Oikawa : Trans. JIM, 12 (1971), 369.

(31) S. Karashima, T. likubo and H. Oikawa : To be published. 


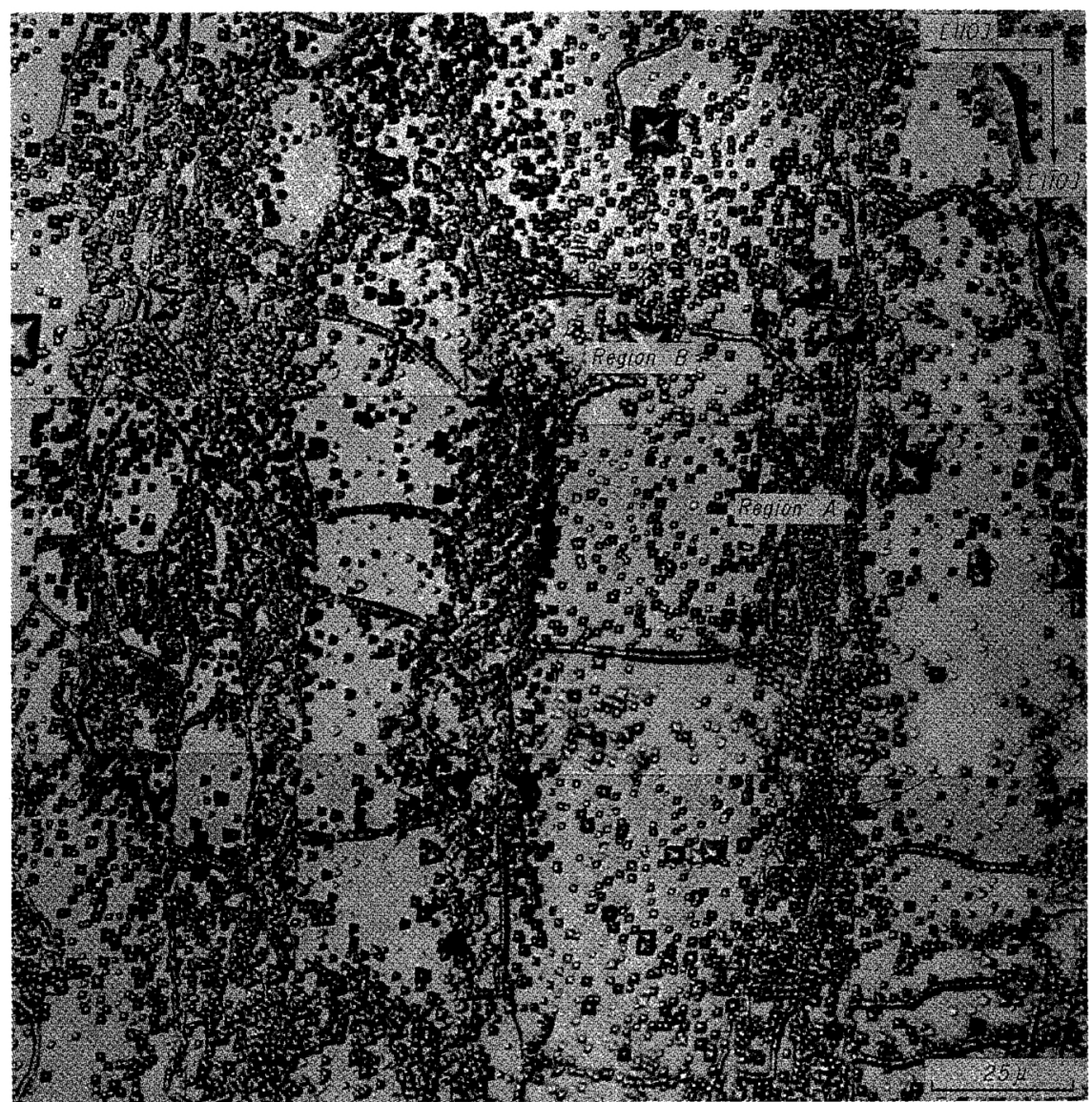

Photo. 3 Substructure on the (001) plane revealed by etch-pit technique after $40 \%$ creep strain at $1.2 \mathrm{~kg} / \mathrm{mm}^{2}$ and $820^{\circ} \mathrm{C}$.

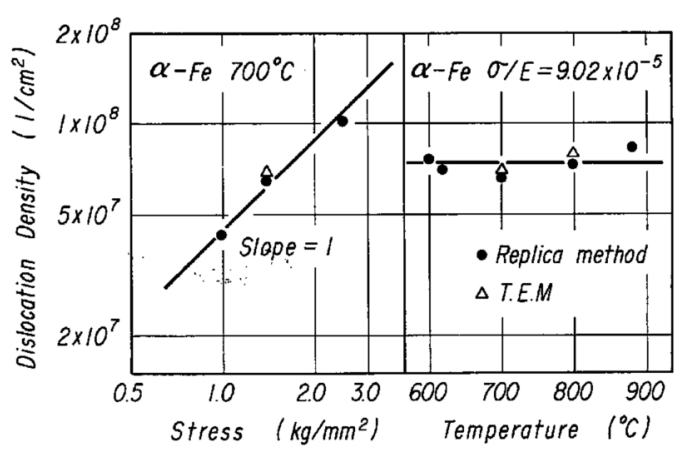

Fig. 6 Dislocation density within subgrains as a function of stress (a) and temperature (b).

further studies are necessary to make clear the origin of the difference.

Hasegawa and Karashima ${ }^{(32)}$ have recently reported that many screw dislocations were observed within subgrains after high-temperature creep of copper single crystals. A similar observation has also been reported in $\mathrm{Fe}-\mathrm{Cr}$ alloys ${ }^{(33)}$. Therefore, the role of screw dislocations in the rate-controlling process of high-temperature creep should not be overlooked hastily.

\section{Summary}

(1) The (001)[110] single crystals showed the usual

(32) T. Hasegawa and S. Karashima : Met. Trans., 1 (1970), 1052.

(33) N. Igata, K. Miyahara and T. Taoka : Proc. Intern. Conf. Electron Microscopy, Stockholm, (1971).

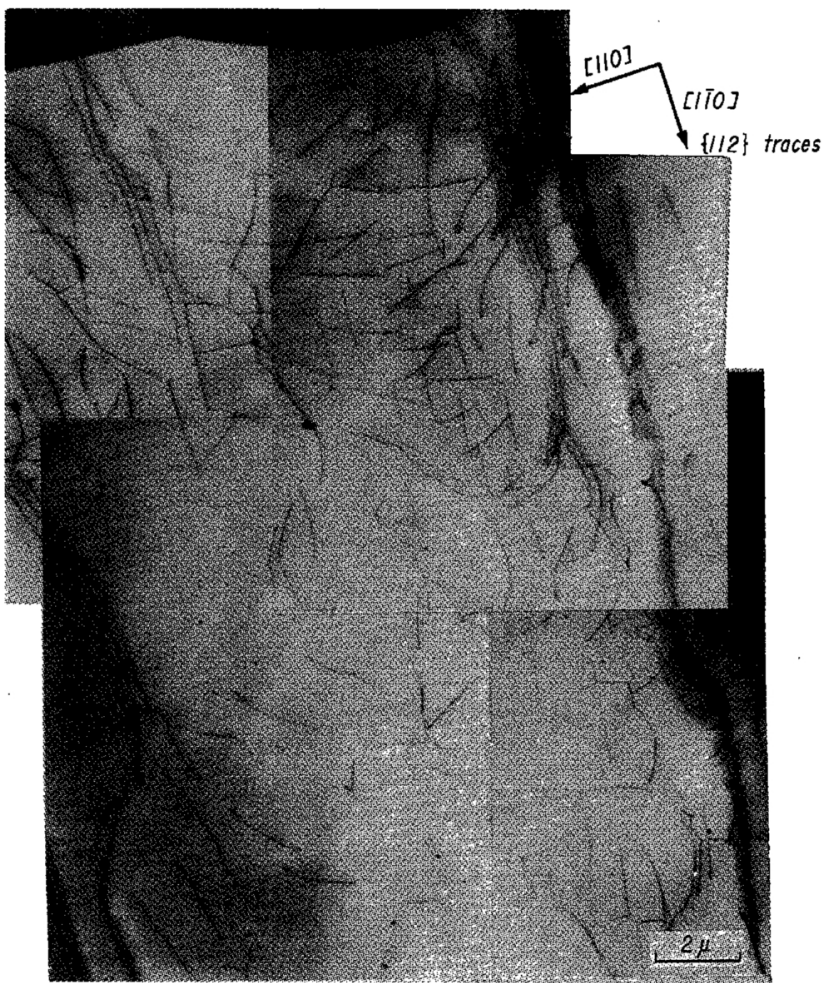

Photo. 4 Transmission electron micrographs showing dislocation substructures in the (001) [110] single crystls after $40 \%$ creep strain at $1.2 \mathrm{~kg} / \mathrm{mm}^{2}$ and $820^{\circ} \mathrm{C}$.

three stages of creep, i.e., transient, steady-state and accelerating stages, at a creep stress larger than 0.7 $\mathrm{kg} / \mathrm{mm}^{2}$. 
(2) Strains up to steady-state stage and up to tertiary stage of the single crystals were larger than those of polycrystalline iron.

(3) Activation energy and activation area for creep obtained on the single crystals were almost the same as those of polycrystalline iron.

(4) Subgrain size and dislocation density within subgrains were independent of creep temperature. Subgrain size was almost inversely proportional to creep stress and the dislocation density within subgrains was proportional to creep stress.
(5) Subgrains were formed during creep as in the case of molybdenum single crystals. However, many screw dislocations were observed within subgrains in the present experiment. The results suggest that creep mechanism due to the motion of screw dislocations should not be hastily discarded.

\section{Acknowledgment}

Thanks are due to Dr. T. Takeuchi, The National Research Institute for Metals, for supplying the iron single crystal sheets. . 